The Astrophysical Journal, 688: L9-L12, 2008 November 20

(C) 2008. The American Astronomical Society. All rights reserved. Printed in U.S.A.

\title{
DISCOVERY OF EXTREME CARBON STARS IN THE LARGE MAGELLANIC CLOUD
}

\author{
R. A. Gruendl, ${ }^{1,2}$ Y.-H. Chu, ${ }^{1,2}$ J. P. Seale, ${ }^{1}$ M. Matsuura, ${ }^{3,4}$ A. K. Speck, ${ }^{5}$ G. C. Sloan, ${ }^{6}$ And L. W. Looney ${ }^{1}$ \\ Received 2008 August 26; accepted 2008 September 26; published 2008 November 4
}

\begin{abstract}
Using Spitzer IRAC and MIPS observations of the Large Magellanic Cloud, we have identified 13 objects that have extremely red mid-IR colors. Follow-up Spitzer IRS observations of seven of these sources reveal varying amounts of $\mathrm{SiC}$ and $\mathrm{C}_{2} \mathrm{H}_{2}$ absorption as well as the presence of a broad $\mathrm{MgS}$ feature in at least two cases, indicating that these are extreme carbon stars. Preliminary estimates find these objects have luminosities of $(4-11) \times 10^{3}$ $L_{\odot}$ and preliminary model fitting gives mass-loss rates between $4 \times 10^{-5}$ and $2 \times 10^{-4} M_{\odot} \mathrm{yr}^{-1}$, higher than any known carbon-rich AGB star in the LMC. These spectral and physical properties require careful reconsideration of dust condensation and mass-loss processes for carbon stars in low-metallicity environments.
\end{abstract}

Subject headings: infrared: stars — Magellanic Clouds — stars: AGB and post-AGB — stars: carbon

\section{INTRODUCTION}

We have undertaken a study of star formation in the Large Magellanic Cloud (LMC; Gruendl \& Chu 2008), using archival Spitzer Space Telescope observations, such as those of Surveying the Agents of a Galaxy's Evolution (SAGE; Meixner et al. 2006). In the process of identifying young stellar objects (YSOs) we noticed 13 bright mid-IR sources (Table 1) that all had similar photometric properties with spectral energy distributions (SEDs) markedly different from those of evolved stars or YSOs: (1) they have extremely red mid-IR colors, [4.5] - [8.0] > 4.0; (2) their SEDs, peaking between 8 and $24 \mu \mathrm{m}$, can be moderately well fit by blackbodies with effective temperatures of $\sim 230-320 \mathrm{~K}$; (3) they all fall in a narrow range of brightness, $7.0>[8.0]>$ 8.5; and (4) none of the sources have counterparts in the Two Micron All Sky Survey Point Source Catalog (2MASS PSC; Skrutskie et al. 2006) or in the Digitized Sky Survey. We dubbed these sources "extremely red objects" (EROs).

The EROs are not likely asteroids because of their high brightnesses and lack of proper motions in SAGE observations from two epochs separated by $\sim 3$ months. They cannot be background galaxies or Galactic sources, as the Spitzer Widearea IR Extragalactic Survey (SWIRE; Lonsdale et al. 2003) and the Galactic Legacy IR Mid-Plane Survey Extraordinaire (GLIMPSE; Benjamin et al. 2003) do not have counterparts with similar mid-IR colors and brightnesses. These EROs are most likely associated with the LMC, and their high luminosities imply that their Galactic counterparts would have saturated in the SWIRE and GLIMPSE Surveys.

Queries of the SIMBAD database found that some EROs had been detected previously in either MSX or IRAS observations (e.g., Schwering 1989). Five have been suggested as possible obscured AGB stars by Loup et al. (1997); however, many of our EROs have also been suggested to be YSO candidates (Whitney et al. 2008). As these EROs have never been confirmed or rejected as

\footnotetext{
${ }^{1}$ Astronomy Department, University of Illinois, 1002 West Green Street, Urbana, IL 61801; gruendl@astro.uiuc.edu, chu@astro.uiuc.edu, seale@ astro.uiuc.edu, lwl@astro.uiuc.edu.

${ }^{2}$ Visiting Astronomer, Cerro Tololo Inter-American Observatory.

${ }^{3}$ National Astronomical Observatory of Japan, Osawa 2-21-1, Mitaka, Tokyo 181-8588, Japan; m.matsuura@nao.ac.jp.

${ }^{4}$ Department of Physics and Astronomy, University College London, Gower Street, London WC1E 6BT, UK.

${ }^{5}$ Department of Physics and Astronomy, University of Missouri, Columbia, MO 65211; speckan@missouri.edu.

${ }^{6}$ Astronomy Department, Cornell University, 610 Space Sciences Building, Ithaca, NY 14853-6801.
}

YSOs spectroscopically, we included seven among our follow-up observations of massive YSOs in the LMC using the Spitzer InfraRed Spectrograph (IRS; Houck et al. 2004). These IRS observations show unambiguously that the EROs are carbon stars; furthermore, the spectra reveal silicon carbide ( $\mathrm{SiC}$ ) absorption features. While $\mathrm{SiC}$ emission features are fairly common in both Galactic and LMC carbon stars, this is the first clear detection of $\mathrm{SiC}$ absorption for LMC carbon stars. Preliminary analysis of the IRS spectra suggests that these are extraordinary carbon stars with very high mass-loss rates. This Letter reports their discovery. In $\S 2$ we describe their basic photometric properties and their possible optical and near-IR counterparts. In $\S 3$ we introduce our IRS observations and in $\S 4$ we discuss the results.

\section{PHOTOMETRIC OBSERVATIONS}

\subsection{Mid-Infrared Observations}

Spitzer observations of the EROs, from SAGE (Meixner et al. 2006), were made with the InfraRed Array Camera (IRAC; Fazio et al. 2004) and the Multiband Imaging Photometer for Spitzer (MIPS; Rieke et al. 2004). Aperture photometry of the EROs was obtained for the IRAC 3.6, 4.5, 5.8, and $8.0 \mu \mathrm{m}$ bands and the MIPS 24 and $70 \mu \mathrm{m}$ bands using the task phot in IRAF. A detailed description of the data reduction, photometric extraction, and uncertainties can be found in Gruendl \& Chu (2008). When multiple observations were available, the photometric measurements were averaged. Table 1 presents the average photometric results: column (1) lists source names that provide epoch J2000.0 coordinates, columns (2)-(7) give flux densities and uncertainties at 3.6, 4.5, 5.8, 8.0, 24, and $70 \mu \mathrm{m}$, and column (8) gives crossidentifications to previously known sources. Figure 1 plots the locations of the EROs in a mid-IR [8.0] versus [4.5] - [8.0] colormagnitude diagram along with other red sources classified as evolved stars by Gruendl \& Chu (2008). The EROs are at the extreme red end of the tail formed by the evolved stars.

\subsection{Complementary Near-Infrared and Optical Photometry}

None of the EROs have a counterpart in the 2MASS PSC. Thus, we obtained deeper near-IR observations at $J$ and $K_{s}$ bands for six of the EROs using the IR Side Port Imager (ISPI; van der Bliek et al. 2004) on the Blanco $4 \mathrm{~m}$ telescope at the Cerro Tololo Inter-American Observatory in 2007 February. The resulting images have a typical effective exposure time of $\sim 300$ and $600 \mathrm{~s}$ in the $J$ and $K_{s}$ bands, respectively, and are flux-calibrated using stars in the 2MASS PSC. The angular 
TABLE 1

Mid-Infrared Photometric Properties and Derived Parameters

\begin{tabular}{|c|c|c|c|c|c|c|c|c|c|}
\hline $\begin{array}{c}\text { Source ID } \\
\text { (1) }\end{array}$ & $\begin{array}{c}m_{3.6} \\
(\mathrm{mag}) \\
(2)\end{array}$ & $\begin{array}{c}m_{4.5} \\
(\mathrm{mag}) \\
(3)\end{array}$ & $\begin{array}{c}m_{5.8} \\
(\mathrm{mag}) \\
(4)\end{array}$ & $\begin{array}{c}m_{8.0} \\
(\mathrm{mag}) \\
(5)\end{array}$ & $\begin{array}{l}m_{24.0} \\
(\mathrm{mag}) \\
(6)\end{array}$ & $\begin{array}{c}m_{70.0} \\
(\mathrm{mag}) \\
(7)\end{array}$ & $\begin{array}{c}\text { Cross } \\
\text { Identification } \\
\text { (8) }\end{array}$ & $\begin{array}{c}L_{\text {bol }} \\
\left(L_{\odot}\right) \\
(9)\end{array}$ & $\begin{array}{c}\text { Mass-Loss } \\
\text { Rate } \\
\left(M_{\odot} \mathrm{yr}^{-1}\right) \\
(10)\end{array}$ \\
\hline $3149-6805358$ & $.92 \pm 0.08$ & $75 \pm 0.05$ & $91 \pm 0.05$ & $7.26 \pm 0.05$ & $3.06 \pm 0.11$ & $2.09 \pm 0.23$ & & 7800 & $1.1 \times 10^{-4}$ \\
\hline 56.7 & $14.92=$ & $2.93=$ & 10.68 & 8.25 & 0.11 & $1.71=$ & IRAS $05036-6649$ & 7000 & $1.5 \times 10^{-4}$ \\
\hline $50405.60-682340.3$ & $16.47 \pm 0.10$ & $13.12 \pm 0.06$ & $10.27 \pm 0.05$ & $7.65 \pm 0.05$ & $3.63 \pm 0.11$ & $>3.32^{\mathrm{a}}$ & IRAS $05042-6827$ & 4950 & $6.3 \times 10^{-5}$ \\
\hline $01.75-6$ & $15.87 \pm 0.08$ & $13.70 \pm 0.06$ & $10.69 \pm 0.06$ & $7.78 \pm 0.06$ & $3.35 \pm 0.11$ & $>1.59^{\mathrm{a}}$ & 051 & 5800 & $8.9 \times 10^{-5}$ \\
\hline 27.0 & $14.97 \pm 0.07$ & $2.26 \pm 0.06$ & $9.89 \pm 0.05$ & $7.61 \pm 0.05$ & $82 \pm 0.11$ & $59 \pm 0.22$ & 33 & 8850 & $1.3 \times 10^{-4}$ \\
\hline 3334.7 & $14.41 \pm 0.07$ & $12.34 \pm 0.06$ & $9.72 \pm 0.05$ & $7.11 \pm 0.05$ & $3.32 \pm 0.11$ & $1.24 \pm 0.24$ & RAS 051 & 7750 & $8.3 \times 10^{-5}$ \\
\hline $052540.63-700827.2$ & $16.02 \pm 0.13$ & $13.20 \pm 0.06$ & $10.45 \pm 0.06$ & $7.80 \pm 0.05$ & $3.82 \pm 0.11$ & $2.58 \pm 0.26$ & IRAS 052 & 4300 & $5.9 \times 10^{-5}$ \\
\hline $2937.89-724952.9$ & $13.61 \pm 0.06$ & $11.14 \pm 0.05$ & $9.02 \pm 0.05$ & $7.09 \pm 0.05$ & $3.81 \pm 0.11$ & $2.92 \pm 0.26$ & & 5550 & $4.2 \times 10^{-5}$ \\
\hline 5 & $347+006$ & $13+0$ & $10.21 \pm 0$ & $7.76 \pm 0$ & $5 \pm 0.11$ & $1.13 \pm 0.22$ & & 9500 & $1.7 \times 10^{-4}$ \\
\hline 209.3 & $14.84 \pm 0.07$ & $11.91 \pm 0$ & $9.40 \pm 0.05$ & $7.11 \pm 0$ & $3.50 \pm 0.11$ & $>2.35^{\mathrm{a}}$ & 43 & 6400 & $6.6 \times 10^{-5}$ \\
\hline $54859.98-703322.5$ & $15.33 \pm 0.08$ & $13.70 \pm 0.06$ & $11.32 \pm 0.06$ & $8.41 \pm 0.06$ & $2.41 \pm 0.11$ & $0.72 \pm 0.22$ & IRAS $05495-7034$ & 11100 & $2.3 \times 10^{-4}$ \\
\hline $055026.08-695603.1$ & $14.26 \pm 0.05$ & $11.81 \pm 0.05$ & $9.25 \pm 0.05$ & $6.94 \pm 0.05$ & $3.10 \pm 0.11$ & $2.36 \pm 0.23$ & IRAS 05509-6956 & 8250 & $9.0 \times 10^{-5}$ \\
\hline $055133.60-711933.9$ & $14.06 \pm 0.06$ & $11.28 \pm 0.05$ & $9.03 \pm 0.05$ & $7.01 \pm 0.05$ & $3.87 \pm 0.11$ & $3.38 \pm 0.26$ & IRAS $05522-7120$ & 5200 & $4.4 \times 10^{-5}$ \\
\hline
\end{tabular}

${ }^{\text {a }}$ For nondetections a $3 \sigma$ upper limit is given.

resolution of the images is typically $\lesssim 1.0^{\prime \prime}$ and the astrometric accuracy is better than $0.2^{\prime \prime}$. For a more detailed description of the near-IR data reduction, see Gruendl \& Chu (2008).

Table 2 presents the near-IR photometry and $3 \sigma$ upper limits for the six EROs observed. Only two EROs, 051301.75-693351.0 and 053044.10-714300.5, have near-IR counterparts projected within $0.2^{\prime \prime}$ as indicated in the last column of the table. To supplement our ISPI observations, we have used the Magellanic Clouds Point Source Catalog from the InfraRed Survey Facility (IRSF; Kato et al. 2007). This survey has an astrometric accuracy of $\sim 0.1^{\prime \prime}$ and $90 \%$ completeness limits of 18.5 and 17.4 for $m_{J}$ and $m_{K}$, respectively. IRSF sources with spatial coincidence of $1^{\prime \prime}$ or better are also included in Table 2. Only two IRSF sources are within $0.2^{\prime \prime}$ or less from their respective EROs and are the same counterparts identified by our ISPI observations. The other three IRSF sources are included for completeness but are unlikely to be near-IR counterparts to the EROs.

To search for optical counterparts, we used the optical pho-

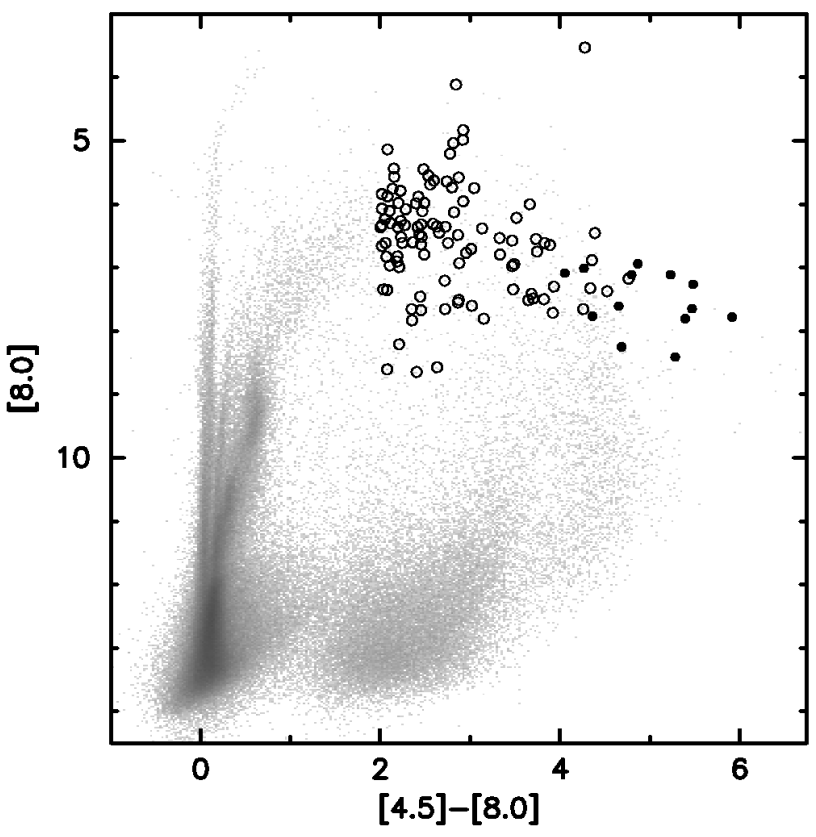

FIG. 1.-Color-magnitude diagram showing IRAC [8.0] vs. [4.5] - [8.0]. The gray-scale Hess diagram shows all data from the LMC. The location of the EROs are plotted with filled circles while other sources classified as evolved stars by Gruendl \& Chu (2008) have been plotted with open circles. tometry from the Magellanic Clouds Photometric Survey (MCPS; Zaritsky et al. 2004) which has an astrometric accuracy of $\sim 0.5^{\prime \prime}$ and is generally complete to $m_{V}=20$. We find that three EROs have faint $\left(m_{V}>19\right)$ optical sources within $1^{\prime \prime}$, but are likely unrelated as none have a spatial coincidence better than $0.7^{\prime \prime}$. In Figure 2 we combine all photometric data for each source to get a broad SED extending from the optical to $70 \mu \mathrm{m}$.

\section{IRS OBSERVATIONS}

Seven EROs were included in our Spitzer IRS survey of YSOs (PID = 40650), which used the Short-Low (SL) modules to obtain spectra over $\sim 5.2-14.5 \mu \mathrm{m}$ with spectral resolution $R(\equiv \delta \lambda / \lambda)$ of 64-128, and the Short-High (SH) and LongHigh (LH) modules to obtain spectra over $\sim 9.9-37.2 \mu \mathrm{m}$ with spectral resolution of $\sim 600$. Background observations with the same integration times were made in nearby regions with low surface brightnesses in the IRAS 12 and $25 \mu \mathrm{m}$ maps.

To extract spectra, we used the basic calibrated data (BCD) from the Spitzer Science Center's pipeline. Rogue pixels and flagged data were cleaned using the IRSCLEAN package (ver. 1.9). Multiple exposures for each slit position were median averaged. The background was then removed in each module by subtracting the cleaned BCD image data at the background position. For the SL modules, an additional local background was subtracted by differencing the two nod positions. Spectra were extracted using the SMART software (Higdon et al. 2004) with a full aperture extraction for the $\mathrm{SH}$

TABLE 2

Possible Near-Infrared Counterparts

\begin{tabular}{cccc}
\hline \hline Source ID & $\begin{array}{c}m_{J} \\
(\mathrm{mag})\end{array}$ & $\begin{array}{c}m_{K} \\
(\mathrm{mag})\end{array}$ & $\begin{array}{c}\text { Offset } \\
(\operatorname{arcsec})\end{array}$ \\
\hline \multicolumn{4}{c}{ ISPI Observations } \\
\hline $050231.49-680535.8$ & $>19.67$ & $>18.63$ \\
$050405.60-682340.3$ & $>19.84$ & $>18.60$ \\
$051301.75-693351.0$ & $16.91 \pm 0.05$ & $16.65 \pm 0.06$ & $<0.2$ \\
$052937.89-724952.9$ & $>19.52$ & $>18.74$ \\
$053044.10-714300.5$ & $17.79 \pm 0.07$ & $16.77 \pm 0.07$ & $<0.2$ \\
$055026.08-695603.1$ & $>19.82$ & $>18.74$ \\
\hline \multicolumn{5}{c}{ Possible Counterparts in IRSF Catalog } \\
\hline $051301.75-693351.0$ & $16.98 \pm 0.02$ & $16.27 \pm 0.06$ & 0.1 \\
$051848.36-693334.7$ & $17.27 \pm 0.03^{\mathrm{a}}$ & $16.66 \pm 0.12^{\mathrm{a}}$ & 1.0 \\
$053044.10-714300.5$ & $18.54 \pm 0.08$ & $17.23 \pm 0.22$ & 0.2 \\
$052540.63-700827.2$ & $17.77 \pm 0.04^{\mathrm{a}}$ & $17.08 \pm 0.17^{\mathrm{a}}$ & 0.7 \\
$054134.73-694209.3$ & $19.61 \pm 0.21^{\mathrm{a}}$ & $16.70 \pm 0.09^{\mathrm{a}}$ & 0.6 \\
\hline
\end{tabular}

${ }^{\mathrm{a}}$ Doubtful counterpart. 


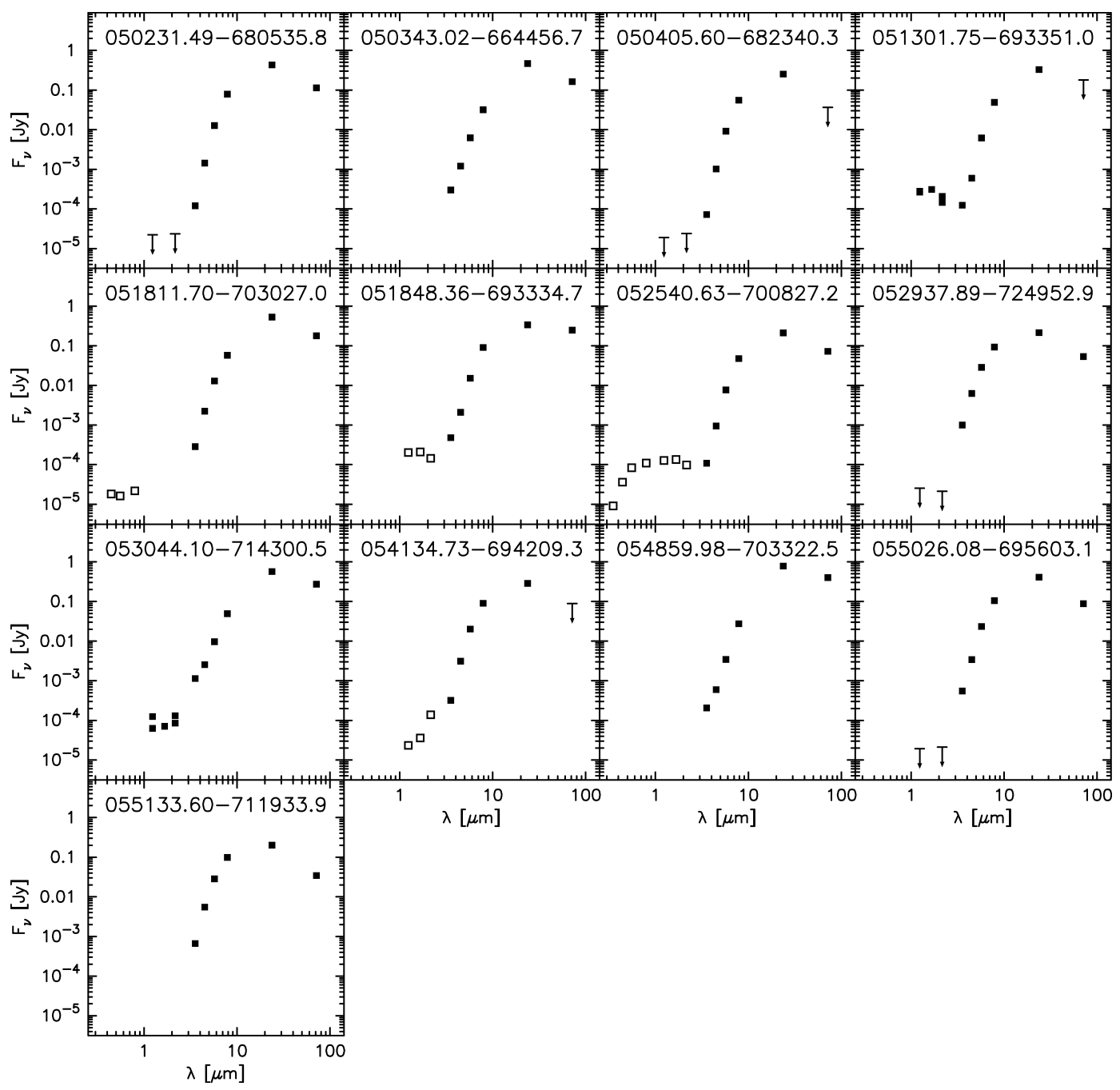

FIG. 2.-The SEDs of the EROs using optical photometry from the MCPS, near-IR photometry from our ISPI observations or the IRSF survey, and mid-IR photometry from IRAC and MIPS measurements. Measurements with good astrometric coincidence are plotted with filled symbols, while those with poorer coincidence are indicated by open symbols. Upper limits for nondetections are shown as arrows with a crossbar at the $3 \sigma$ significance limit.

and LH modules and the tapered column point source method for the SL modules. The SH and LH spectra were automatically defringed using the IRSFRINGE package (ver. 1.1). To combine all spectra, we used the SL extracted spectra to set the flux level (as their background subtraction was more robust) and then applied a multiplicative scale factor for the $\mathrm{SH}$ and $\mathrm{LH}$ spectra based on the continuum where the spectra overlapped. The resulting spectra span the wavelength range from 5.2 to $37.2 \mu \mathrm{m}$. A more detailed description is presented in J. P. Seale et al. (2009, in preparation) which reports the results of our IRS survey of LMC YSO candidates.

\section{RESULTS}

Figure 3 presents the IRS spectra of seven EROs along with the IRAC and MIPS mid-IR photometry. Clearly, these spectra are dominated by dust continuum emission. Interestingly, the $11.3 \mu \mathrm{m}$ $\mathrm{SiC}$ feature is detected in absorption in nearly all spectra; in Figure 3 the spectra have been ordered from top to bottom based on the strength of the $\mathrm{SiC}$ absorption feature. In addition, the $\mathrm{C}_{2} \mathrm{H}_{2}$ (acetylene) $13.7 \mu \mathrm{m}$ absorption feature appears in every spectrum, and the $\mathrm{C}_{2} \mathrm{H}_{2} 7.5 \mu \mathrm{m}$ absorption feature appears in some spectra. In contrast, the $\mathrm{MgS}$ emission feature at $\sim 26 \mu \mathrm{m}$ is clearly detected in the spectrum of $055026.08-695603.1$ and possibly in that of 052937.89-724952.9. Finally, we note that the only source without SiC absorption, 051811.70-703027.0, has a nearly featureless spec- trum similar to AFGL 618 (the Westbrook Nebula) a carbon-rich proto-planetary nebula (Kraemer et al. 2002; Cernicharo et al. 2001a, 2001b).

\subsection{Mass-Loss Rates}

The extreme red colors of these EROs, [8.0] $-[24]=3-5$, suggest that they have high opacities and hence high mass-loss rates, $\dot{M}$. Simple fits to the mid-IR SEDs of the EROs give bolometric luminosities in the range of (4-11) $\times 10^{3} L_{\odot}$ (for a distance of 50 kpc; Feast 1999). Preliminary analysis using the radiative transfer model DUSTY (Ivezic \& Elitzur 1995) shows that the highest optical depths $(\tau)$ are found for $054859.98-703322.5$, with $\tau$ 's of 270, 8.1, and 10.0 at 1,10 , and $11.3 \mu \mathrm{m}$, respectively. Assuming a gas-todust ratio of 200, the estimated $\dot{M}$ are in the range of (0.4-2.3) $\times$ $10^{-4} M_{\odot} \mathrm{yr}^{-1}$ (see Table 1 ). We have adjusted values of $\dot{M}$ from other works to reflect a gas-to-dust ratio of 200 .

Our derived values for $\dot{M}$ are higher than previously known for carbon-rich AGB stars in the LMC. Furthermore, these 13 sources more than double the previously known eight carbon-rich AGB stars in the LMC with $\dot{M}$ higher than $10^{-5} M_{\odot} \mathrm{yr}^{-1}$ (van Loon et al. 1999; Groenewegen et al. 2007). More intriguingly, these sources typically exceed the maximum $\dot{M}$ expected for both oxygen-rich and carbon-rich AGB stars (van Loon et al. 1999). For example, the $\dot{M}$ of $054859.98-703322.5,2.3 \times 10^{-4} M_{\odot} \mathrm{yr}^{-1}$, is much 


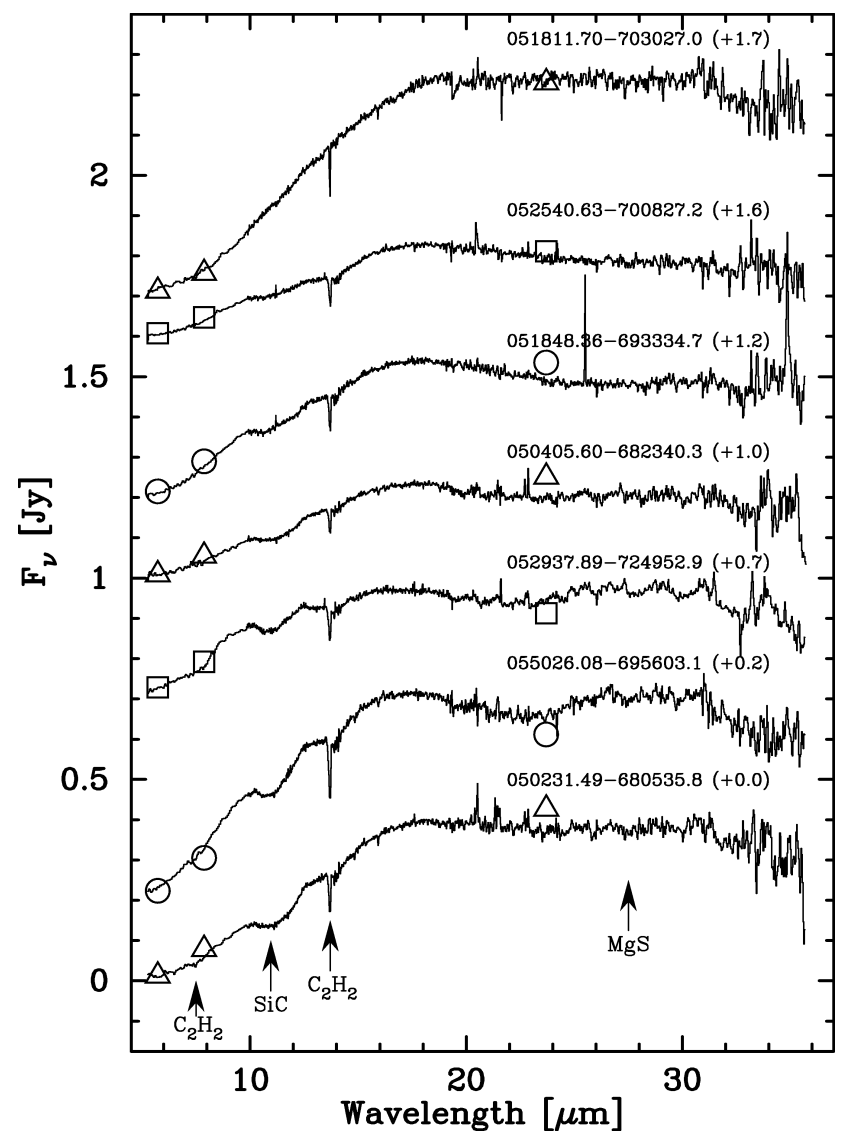

FIG. 3.-IRS spectra for seven EROs. The spectra are roughly ordered from top to bottom based on the increasing strength of the $\mathrm{SiC}$ feature at $11.3 \mu \mathrm{m}$. Each spectrum has been offset by a constant value in Jy which is given after the source name in the figure. Our mid-IR photometry for each source are plotted with each spectrum for comparison using alternating symbols.

higher than the maximum expected for its bolometric luminosity, $4 \times 10^{-5} M_{\odot} \mathrm{yr}^{-1}$.

Comparisons of carbon stars in the Magellanic Clouds and the Galaxy show that $\dot{M}$ does not depend on metallicity (Matsuura et al. 2005; Sloan et al. 2008), because $\dot{M}$ depends on the amount of carbon available in the atmosphere, which is ultimately synthesized within these stars. These new extreme carbon stars show that the highest $\dot{M}$ found in the LMC are comparable to those of their Galactic counterparts (e.g., Speck et al. 2008). The mean bolometric luminosity of the EROs, $\sim 7100 L_{\odot}$, corresponds to main-sequence masses of 1.5-2.5 $M_{\odot}$ (using Fig. 20 in Vassiliadis \& Wood 1993), where the lower limit is set by the mass range to become carbon stars $\left(1.5-5.0 M_{\odot}\right)$. It is puzzling that such lowmass stars could reach the highest $\dot{M}$. This might be caused by some specific combinations of abundance and pressure-temperature profile in the stellar atmosphere, resulting in an unusual condensation sequence (Lodders \& Fegley 1995). It is also possible that a carbon-rich dust disk is responsible for the $\mathrm{SiC}$ absorption then the $\dot{M}$ based on a spherical symmetry is not valid. These possibilities will be considered in greater detail in future work.

\subsection{Spectral Features}

The occurrence of $\mathrm{SiC}$ absorption features is surprising. These features are rare in Galactic extreme carbon stars, with only two unambiguous detections (Speck et al. 1997) and another eight more tentative detections (Pitman et al. 2006; Speck et al. 2008). This raises the question of why the $\mathrm{SiC}$ absorption feature is seemingly common in the LMC extreme carbon stars.

Recent Spitzer observations show that planetary nebulae (PNe) in the LMC often show strong $\mathrm{SiC}$ emission (Stanghellini et al. 2007; J. Bernard-Salas et al. 2008, in preparation), while in Galactic $\mathrm{PNe}$, it is generally weak (Barlow 1983; Rinehart et al. 2002). The presence of $\mathrm{SiC}$ features in EROs and PNe in the LMC suggests that (1) SiC grains are formed more abundantly in the LMC than previous recognized (e.g., Zijlstra et al. 2006); and (2) the LMC metallicity allows $\mathrm{SiC}$ to form and survive in the late AGB phase and into the PN phase more efficiently than in the Galaxy. It has been hypothesized by Leisenring et al. (2008) that $\mathrm{MgS}$ tends to form as a mantle on preexisting $\mathrm{SiC}$ grains and gives rise to the emission feature at $30 \mu \mathrm{m}$. This hypothesis is supported by a recent study of the correlation between mass-loss rate and the amount of $\mathrm{SiC}$ required to model Galactic extreme carbon star spectra (Speck et al. 2008). Only two of the LMC EROs show evidence for a MgS feature at $30 \mu \mathrm{m}$ indicating that the $\mathrm{SiC}$ grains may not be coated for some of these objects. Current models for the effect of metallicity on condensation sequences in the Galaxy and the Magellanic Clouds (e.g., Lagadec et al. 2007; Leisenring et al. 2008; Speck et al. 2006) need to be reanalyzed in light of this discovery.

This research was supported by NASA grants JPL1264494 and JPL1290956. We thank S. D. Points for obtaining the ISPI observations used in this Letter. This research has made use of the SIMBAD database, operated at CDS, Strasbourg, France.

\section{REFERENCES}

Barlow, M. J. 1983, in IAU Symp. 103, Planetary Nebulae, ed. D. R. Flower (Dordrecht: Reidel), 105

Benjamin, R. A., et al. 2003, PASP, 115, 953

Cernicharo, J., et al. 2001a, ApJ, 546, L123

- 2001b, ApJ, 546, L127

Fazio, G., et al. 2004, ApJS, 154, 10

Feast, M. 1999, in IAU Symp. 190, New Views of the Magellanic Clouds,

ed. Y.-H. Chu et al. (San Francisco: ASP), 542

Groenewegen, M. A. T., et al. 2007, MNRAS, 376, 313

Gruendl, R. A., \& Chu, Y.-H. 2008, ApJS, submitted

Higdon, S. J. U., et al. 2004, PASP, 116, 975

Houck, J. R., et al. 2004, ApJS, 154, 18

Ivezic, Z., \& Elitzur, M. 1995, ApJ, 445, 415

Kato, D., et al. 2007, PASJ, 59, 615

Kraemer, K. E., et al. 2002, ApJS, 140, 389

Lagadec, E., et al. 2007, MNRAS, 376, 1270

Leisenring, J. M., Kemper, F., \& Sloan, G. C. 2008, ApJ, 681, 1557

Lodders, K., \& Fegley, B., Jr. 1995, Meteoritics, 30, 661

Lonsdale, C. J., et al. 2003, PASP, 115, 897

Loup, C., et al. 1997, A\&AS, 125, 419
Matsuura, M., et al. 2005, A\&A, 434, 691

Meixner, M., et al. 2006, AJ, 132, 2268

Pitman, K. M., Speck, A. K., \& Hofmeister, A. M. 2006, MNRAS, 371, 1744

Rieke, G. H., et al. 2004, ApJS, 154, 25

Rinehart, S. A., et al. 2002, MNRAS, 336, 66

Schwering, P. B. W. 1989, A\&AS, 79, 105

Skrutskie, M. F., et al. 2006, AJ, 131, 1163

Sloan, G. C., et al. 2008, preprint (arXiv:0807.2998)

Speck, A. K., Barlow, M. J., \& Skinner, C. J. 1997, MNRAS, 288, 431

Speck, A. K., Corman, A. B., Wheeler, C. H., Wakeman, K., \& Thompson, G. D. 2008, ApJ, submitted

Speck, A. K., et al. 2006, ApJ, 650, 892

Stanghellini, L., et al. 2007, ApJ, 671, 1669

van der Bliek, N. S., et al. 2004, Proc. SPIE, 5492, 1582

van Loon, J. Th., et al. 1999, A\&A, 351, 559

Vassiliadis, E., \& Wood, P. R. 1993, ApJ, 413, 641

Whitney, B. A., et al. 2008, AJ, 136, 18

Zaritsky, D., Harris, J., Thompson, I. B., \& Grebel, E. K. 2004, AJ, 128, 1606

Zijlstra, A., et al. 2006, MNRAS, 370, 1961 